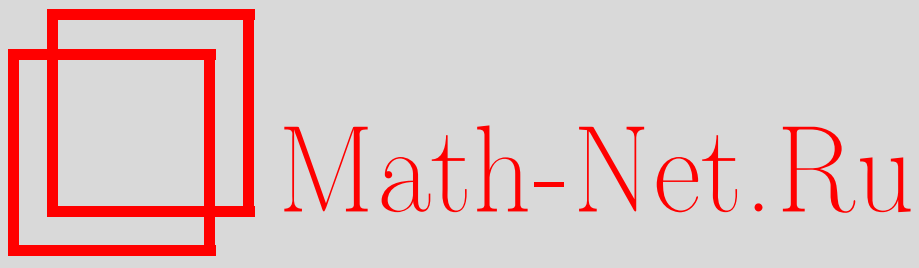

Ю. И. Кадашевич, С. П. Помыткин, А. М. Пейсахов, Влияние формы задания градиента деформаций на решение задач эндохронной теории непругости при конечных деформациях, Вестн. Сам. гос. техн. ун-та. Сер. Физ.-мат. науки, 2006, выпуск 42, 82-85

DOI: https://doi.org/10.14498/vsgtu415

Использование Общероссийского математического портала Math-Net.Ru подразумевает, что вы прочитали и согласны с пользовательским соглашением http://www . mathnet.ru/rus/agreement

Параметры загрузки:

IP: 107.22 .136 .117

26 апреля 2023 г., 08:31:54

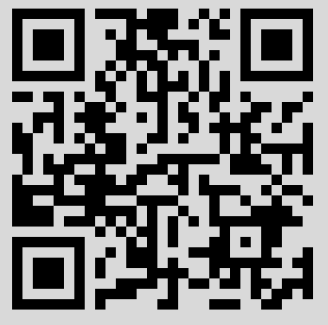


УДК 539.374

Ю.И.Кадашевич, С.П.Помыткин, А.М.Пейсахов

\section{ВЛИЯНИЕ ФОРМЫ ЗАДАНИЯ ГРАДИЕНТА ДЕФОРМАЦИЙ НА РЕШЕНИЕ ЗАДАЧ ЭНДОХРОННОЙ ТЕОРИИ НЕПРУГОСТИ ПРИ КОНЕЧНЫХ ДЕФОРМАЦИЯХ}

В рамках эндохронной теории неупругости, учитывающей конечные деформации, анализируется влияние формы градиента деформаций и начальных условий для его компонент на тип решения задач жесткого нагружения.

В работе [1] авторы предложили новый подход к построению теории ползучести эндохронного типа, учитывающей конечные деформации. В предлагаемой работе дается расширение теории, которое позволяет описать ряд экспериментальных данных, опубликованных в литературе.

Исследуется основной вариант эндохронной теории неупругости для конечных деформаций в виде:

$$
\begin{aligned}
& \alpha \tau \stackrel{*}{\sigma}+\sigma|\dot{r}|=2 G\left(\tau \stackrel{*}{r}+\frac{r}{g+\alpha}|\dot{r}|\right), \\
& \varepsilon^{*}=D, \Omega=\dot{Q} Q^{T}, \\
& \dot{\varepsilon}+\varepsilon \Omega-\Omega \varepsilon={ }_{\varepsilon}^{*}, \dot{\sigma}+\sigma \Omega-\Omega \sigma={ }^{*}, \\
& r=\varepsilon-(1-\alpha) \frac{\sigma}{2 G}, \\
& \tau=\tau(|r|,|\dot{r}|), \quad|\dot{r}|=\sqrt{\frac{d r}{d t}: \frac{d r}{d t}} .
\end{aligned}
$$

В соотношения (1) в безындексной форме записи входят: $\alpha$ - параметр эндохронности, $\tau$ аналог деформационного предела текучести, $\sigma, \varepsilon, r$ - девиаторы тензоров напряжений, деформаций и вспомогательного параметрического тензора, $G$ - модуль сдвига, $g$ - аналог коэффициента упрочнения, $Q$ - ортогональный тензор поворота, $\Omega$ - спин, $D$ - тензор скоростей деформаций. Кроме того, здесь используется, что $\varepsilon_{i i}=\sigma_{i i} / K, D=\left(L+L^{T}\right) / 2, L=\dot{F} F^{-1}$, $Q=F u^{-1}$ - полярное разложение ортогонального тензора поворота, $F$ - градиент деформаций; $L$ - скорость градиента деформаций, $u$ - правый тензор удлинения, $K$ - объемный модуль.

При постановке и решении задач сложного нагружения необходимо четко сформулировать вид градиента деформаций $F$, его начальные значения и форму ортогонального тензора поворота $Q$. В работе [1] был рассмотрен вариант теории, в котором градиент деформации имел вид:

$$
\begin{gathered}
F=\left(\begin{array}{ccc}
k_{11} & 0 & 0 \\
k_{21} & k_{22} & 0 \\
k_{31} & k_{32} & k_{33}
\end{array}\right), \\
k_{11}(0)=k_{22}(0)=k_{33}(0)=1, k_{21}(0)=k_{31}(0)=k_{32}(0)=0 .
\end{gathered}
$$

Предположим теперь, что форма градиента деформаций имеет структуру

$$
F=\left(\begin{array}{ccc}
k_{11} & m_{1} k_{21} & m_{2} k_{31} \\
k_{21} & k_{22} & m_{3} k_{32} \\
k_{31} & k_{32} & k_{33}
\end{array}\right),
$$

где $m_{1}, m_{2}, m_{3}$ - постоянные параметры, характеризующие исходные свойства материала, и рассмотрим два примера, которые позволяют оценить новые возможности теории.

Предположим, что градиент деформаций таков, что 


$$
F=\left(\begin{array}{ccc}
a_{1} & m b & 0 \\
b & a_{2} & 0 \\
0 & 0 & a_{3}
\end{array}\right), \Delta=a_{1} a_{2}-m b^{2}, F^{-1}=\frac{1}{\Delta}\left(\begin{array}{ccc}
a_{2} & -m b & 0 \\
-b & a_{1} & 0 \\
0 & 0 & \Delta / a_{3}
\end{array}\right),
$$

тогда ортогональный тензор поворота будет иметь структуру:

Легко проверить, что в этом случае

$$
Q=\left(\begin{array}{ccc}
\cos \beta & \sin \beta & 0 \\
-\sin \beta & \cos \beta & 0 \\
0 & 0 & 1
\end{array}\right)
$$

$$
\begin{gathered}
\dot{a_{1}} a_{2}-m b \dot{b}=\Delta \cdot D_{11}, \dot{a}_{2} a_{1}-m b \dot{b}=\Delta \cdot D_{22}, D_{23}=D_{13}=0, \\
\frac{\dot{a_{3}}}{a_{3}}=D_{33}, \dot{b}\left(a_{2}+m a_{1}\right)-b\left(\dot{a_{2}}+m \dot{a_{1}}\right)=2 \cdot \Delta \cdot D_{12} .
\end{gathered}
$$

Кроме того, $\operatorname{tg} \beta=\frac{b(m-1)}{a_{1}+a_{2}}$. В этом классе градиентов решим две задачи (по заданным компонентам тензора скоростей деформации восстанавливаются значения компонент градиента деформаций и компонент ортогонального тензора поворота).

Задача 1. Предположим, что

$$
D_{11}=D_{22}=D_{33}=D_{13}=D_{23}=0, D_{12}=1, m \neq 0, a_{1}(0)=1, a_{2}(0)=1, a_{3}(0)=1, b(0)=0 .
$$

Тогда $a_{1}=a_{2}, a_{1}^{2}=m b^{2}+1, a_{3}=1$. Нетрудно проверить, что при $m=k^{2}$, задача имеет решение:

$$
\begin{gathered}
a_{1}=\operatorname{ch}\left(A_{1} t\right), b=\frac{1}{k} \operatorname{sh}\left(A_{1} t\right), \operatorname{tg} \beta=B_{1} \cdot \operatorname{th}\left(A_{1} t\right), \dot{\beta}=\frac{A_{1} B_{1}}{\operatorname{ch}^{2}\left(A_{1} t\right)+B_{1}^{2} \operatorname{sh}^{2}\left(A_{1} t\right)}, \\
A_{1}=\frac{2 k}{1+k^{2}}, B_{1}=\frac{k^{2}-1}{2 k} .
\end{gathered}
$$

Если же $m=-l^{2}$, тогда

$$
\begin{gathered}
a_{1}=\cos \left(A_{2} t\right), b=\frac{1}{l} \sin \left(A_{2} t\right), \operatorname{tg} \beta=B_{2} \cdot \operatorname{tg}\left(A_{2} t\right), \dot{\beta}=\frac{A_{2} B_{2}}{\cos ^{2}\left(A_{2} t\right)+B_{2}^{2} \sin ^{2}\left(A_{2} t\right)}, \\
A_{2}=\frac{2 l}{1-l^{2}}, B_{2}=\frac{-\left(1+l^{2}\right)}{2 l} .
\end{gathered}
$$

Отметим, что аналогичная задача (определение угла $\beta$ ) исследовалась и в работе [2]. В отличие от авторов данной публикации, в этой работе задавалась не форма градиента деформаций, а связь спина со скоростью тензора деформаций. Качественное поведение угла $\beta$ в условиях сдвиговых деформаций оказалось сходным (при этом тождественными результаты были лишь в одном случае, когда параметр $m=3$, а параметр $\eta$, рассматриваемый в работе [2], paвен 2).

После нахождения величин $a_{1}, a_{2}, a_{3}$ и $b$ по соотношениям (1) определяются тензор деформаций $\varepsilon$ и тензор напряжений $\sigma$. На рис. $1 a, 1 \sigma$ и 1 в приведены результаты расчетов для значений параметра: $m=2, m=0,5$ и $m=-0,6$. С качественной точки зрения результаты отвечают работам [2-4]. Для простоты при расчетах принято, что $\alpha=1, \tau=1,2 G=1, g=\infty$.

Задача 2. Пусть теперь $D_{12}=0, D_{23}=0, D_{13}=0, D_{11}=1, D_{22}=D_{33}=-0,5$. В этом случае можно установить, что $b=\left(a_{2}+m a_{1}\right) \cdot C_{0}$, где $C_{0}=$ const .

Для простоты примем, что $C_{0}=1, a_{1}(0)=1, a_{2}(0)=1$, тогда $b(0)=1+m$ и $b=a_{2}+m a_{1}$. Значения $a_{1}$ и $a_{2}$ определяются решениями уравнения

$$
\ddot{a}-\left(D_{11}+D_{22}\right) \dot{a}+\left(D_{3}^{2} m^{2}+D_{11} D_{22}\right) a=0,
$$

где $D_{3}=D_{22}-D_{11}$.

Кроме того, справедливы следующие соотношения:

$$
\dot{a_{1}}=D_{11} a_{1}+m b D_{3}, \dot{a_{2}}=D_{22} a_{2}-m^{2} b D_{3} .
$$




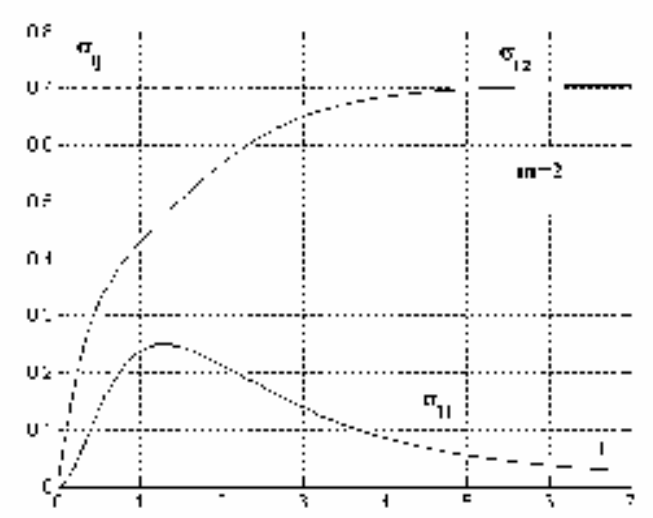

$a$

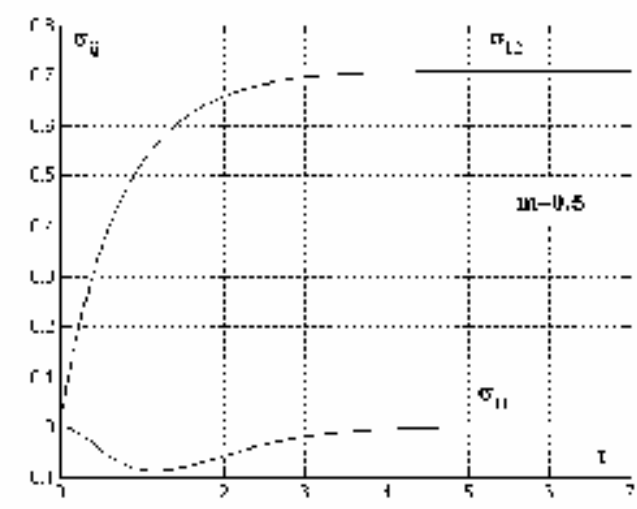

$\sigma$

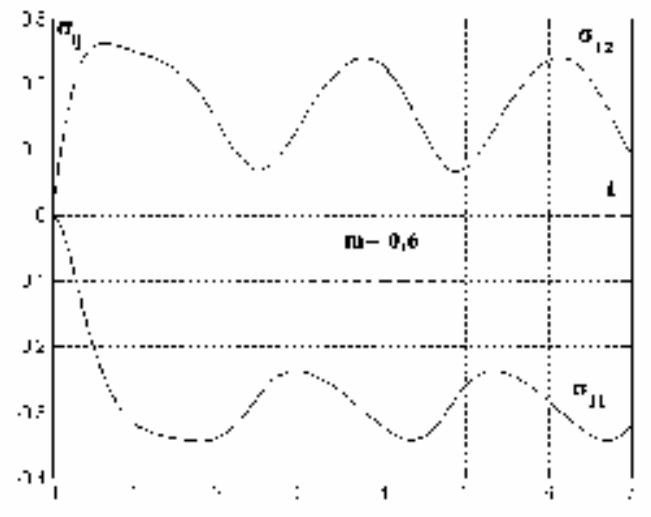

B

Р и с. 1.

Можно установить, что при $|m| \leq 0,5$, решение носит монотонный характер, а при $|m|>0,5$ - колебательный. В частности, для изохорического растяжения (случай, когда $D_{11}=1$ и $\left.D_{22}=D_{33}=-0,5\right)$, определяющее уравнение для $а$ принимает вид: $\ddot{a}-0,5 \dot{a}+\left(\frac{9}{4} m^{2}-\frac{1}{2}\right) a=0$ с начальными условиями $a_{1}(0)=1, \dot{a}_{1}(0)=1-\frac{3}{2} m(m+1), a_{2}(0)=1, \dot{a}_{2}(0)=-\frac{1}{2}+\frac{3}{2} m^{2}(m+1)$.

Пусть $k_{1}=0,25+\omega, k_{2}=0,25-\omega, \omega=0,75 \sqrt{1-4 m^{2}}$, тогда при $|m|<0,5$ решение выражается соотношениями

$$
\begin{aligned}
& a_{1}=C_{1}\left(e^{k_{1} t}-e^{k_{2} t}\right)+e^{k_{2} t}, C_{1}=\frac{\dot{a}_{1}(0)-k_{2}}{2 \omega}, \\
& a_{2}=C_{2}\left(e^{k_{1} t}-e^{k_{2} t}\right)+e^{k_{2} t}, C_{2}=\frac{\dot{a_{2}}(0)-k_{2}}{2 \omega} .
\end{aligned}
$$

Если $|m|>0,5$ то структура решения имеет вид $\left(\omega=0,75 \sqrt{4 m^{2}-1}\right)$ :

$$
\begin{aligned}
& a_{1}=e^{0,25 t}\left(\cos \omega t+C_{1} \sin \omega t\right), C_{1}=\frac{\dot{a}_{1}(0)-0,25}{\omega}, \\
& a_{2}=e^{0,25 t}\left(\cos \omega t+C_{2} \sin \omega t\right), C_{2}=\frac{\dot{a}_{2}(0)-0,25}{\omega} .
\end{aligned}
$$

Если обозначить отношение $a_{1}$ к $a_{2}$ через $z$, то 


$$
\begin{gathered}
\operatorname{tg} \beta=M=\frac{(m-1)(1+m z)}{1+z} \text { и } \\
\dot{\beta}=\frac{2(m-1)^{2}\left(C_{1}-C_{2}\right) \omega e^{2 \omega t}}{\left(1+M^{2}\right)(1+z)^{2}\left(C_{2} e^{2 \omega t}+1-C_{2}\right)^{2}}, \text { если }|m|<0,5, \\
\dot{\beta}=\frac{(m-1)^{2}\left(C_{1}-C_{2}\right)}{\left(1+M^{2}\right)(1+z)^{2}\left(\cos \omega t+C_{2} \sin \omega t\right)^{2}}, \text { если }|m|>0,5 .
\end{gathered}
$$

Для выявления качественного поведения решения было принято, что $\alpha=1, \tau=1,2 G=1$, $g=\infty$ и рассмотрены рабочие уравнения для напряжений в задаче изохорического растяжения в случае, когда $D_{11}=1, D_{22}=D_{33}-0,5, \sigma_{22}=\sigma_{33}=-0,5 \sigma_{11}$, в форме

$$
\dot{\sigma}_{11}+\sqrt{\frac{3}{2}} \sigma_{11}-2 \dot{\beta} \sigma_{12}=1, \dot{\sigma_{12}}+\sqrt{\frac{3}{2}} \sigma_{12}+\frac{3}{2} \dot{\beta} \sigma_{11}=0 \text {. }
$$

На рис. $2 a$ и $2 \sigma$ приведены графики напряжений $\sigma_{11}$ и $\sigma_{12}$, полученные при решении поставленной задачи для двух значений параметров $m: m=0,1$ и $m=-1,0$.

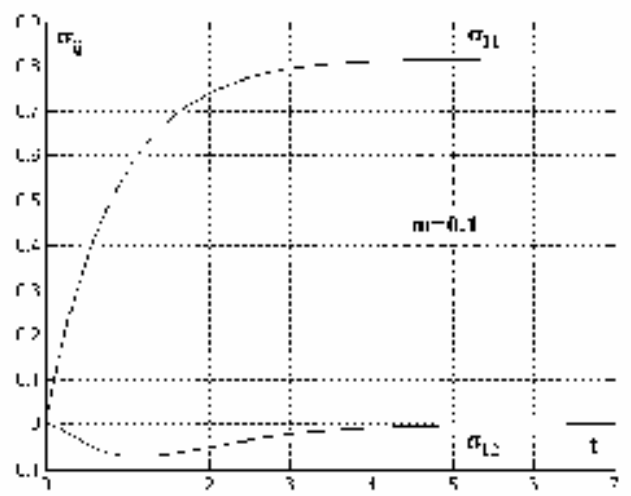

$a$

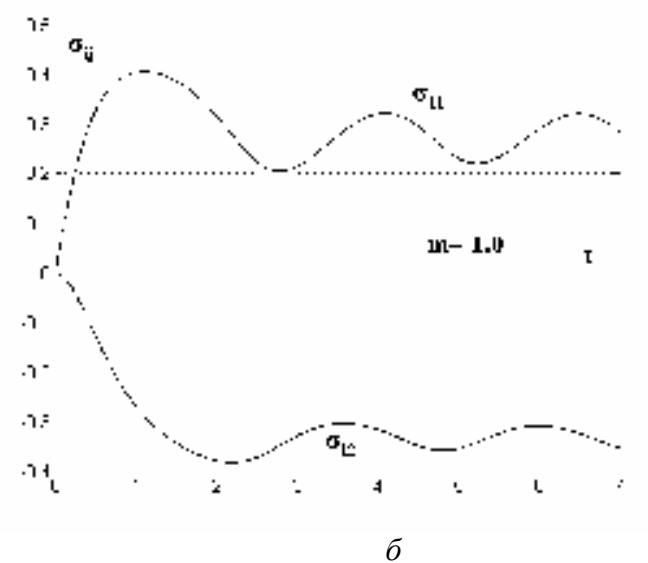

6

Р и с. 2.

Подчеркнем, что внимание к двум поставленным задачам впервые привлекли теоретические работы [2] и [5]. Интересные опытные данные опубликованы в [3, 4, 6].

Таким образом, обобщение эндохронной теории неупругости позволяет существенно расширить описательные возможности теории при изучении простого и сложного нагружений, в соответствии с эффектами, обнаруженными в $[3,4,6]$.

\section{БИБЛИОГРАФИЧЕСКИЙ СПИСОК}

1. Кадашевич Ю.И., Помыткин С.П. Эндохронная теория ползучести при конечных деформациях // Вестник Самарского государственного технического университета. Сер.: Физ.-матем. науки. Самара. 2004. № С.1-7.

2. Dafalias Y.F., Rashid M.M. The effect of plastic spin on anisotropic material behavior // International Journal of Plastisity. 1989. Vol.5. P.227-246.

3. Montheillet F., Cohen M., Jonas J.J. Axial stresses and texture development during the torsion testing of $\mathrm{Al}, \mathrm{Cu}, \alpha$ - $\mathrm{Fe} / /$ Acta Metallurgica. 1984. Vol. 32. P. 2077-2089.

4. Montheillet F., Gilormini P., Jonas J.J. Relation between axial stresses and texture development during the torsion testing: A. Simplified theory. // Acta Metallurgica. 1985. Vol. 33. P. 705-714.

5. Rubin M.B. Plasticity theory formulated in terms of physically based microstructural variables - Part II. Examples // International Journal of Solids and Structures. 1994. Vol.31. N 19. P.2635-2652.

6. Kuroda M., Tvergaard V. Plastic spin associated with a non-normality theory of plasticity // European Journal of Mechanics. A. 2001. Vol.20. N 6. P.893-905.

Работа выполнена при поддержке Российского фонда фундаментальных исследований (грант 05-01-00169). 